\title{
Changing-sign bubble solutions for an anisotropic sinh-Poisson equation
}

\author{
Long Wei
}

Abstract. We consider the following anisotropic sinh-Poisson equation

$$
\operatorname{div}(a(x) \nabla u)+2 \varepsilon^{2} a(x) \sinh u=0 \quad \text { in } \Omega, \quad u=0 \text { on } \partial \Omega,
$$

where $\Omega \subset \mathbb{R}^{2}$ is a bounded smooth domain and $a(x)$ is a positive smooth function. We investigate the effect of anisotropic coefficient $a(x)$ on the existence of bubbling solutions. We show that there exists a family of solutions $u_{\varepsilon}$ concentrating positively and negatively at $\bar{x}$, a given local critical point of $a(x)$, for $\varepsilon$ sufficiently small, for which with the property

$$
2 \varepsilon^{2} a(x) \sinh u_{\varepsilon} \rightarrow 8 \pi \sum_{j=1}^{m} b_{j} \delta_{\bar{x}},
$$

where $b_{j}= \pm 1$. This result shows a striking difference with the isotropic case $(a(x) \equiv$ Constant) in Bartolucci and Pistoia (IMA J Appl Math 72(6):706-729, 2007), Jost et al. (Calc Var Partial Differ Equ 31:263-276, 2008) and Esposito and Wei (Calc Var Partial Differ Equ 34:341-375, 2009).

Mathematics Subject Classification (2000). 35J20, 35J65.

Keywords. Non-simple blow-up solutions, sinh-Poisson equation.

\section{Introduction}

We consider the following generalized sinh-Poisson equation

$$
\operatorname{div}(a(x) \nabla u)+2 \varepsilon^{2} a(x) \sinh u=0 \quad \text { in } \Omega, \quad u=0 \text { on } \partial \Omega,
$$

where $\Omega \subset \mathbb{R}^{2}$ is a bounded smooth domain, $\varepsilon>0$ and $a(x)$ is a smooth function over $\Omega$ satisfying $0<a_{1} \leq a(x) \leq a_{2}<+\infty$.

Equation (1) was motivated by the study of the following classical sinhPoisson equation

$$
\Delta u+2 \varepsilon^{2} \sinh u=0 \text { in } \Omega
$$


where $\Omega \subset \mathbb{R}^{N}$ is a bounded and smooth domain. Problem (2) relates to various dynamics of vorticity with respect to geophysical flows, rotating and stratified fluids and fluid layers excited by electromagnetic forces (see $[10,14,16]$ and the references therein) and constant mean curvature surfaces studied by many works, see $[15,19,23]$ and the references therein.

Recently, the asymptotic behavior of solutions to (2) has been studied on a closed Riemann surface in [17] and [15] and the authors applied so-called "symmetrization method" and "Pohozaev identity" respectively to show that there possibly exist two different types of blow-up for a family of solutions to (2). Moreover, in [15] the authors have proved that if one sets

$$
m_{1}(p)=\lim _{r \rightarrow 0} \lim _{\varepsilon \rightarrow 0} \int_{B_{r}(p)} \varepsilon^{2} e^{u_{\varepsilon}} d v \text { and } m_{2}(p)=\lim _{r \rightarrow 0} \lim _{\varepsilon \rightarrow 0} \int_{B_{r}(p)} \varepsilon^{2} e^{-u_{\varepsilon}} d v \text {, }
$$

then $m_{1}$ and $m_{2}$ are multiplies of $8 \pi$, and

$$
\left(m_{1}(p), m_{2}(p)\right)=8 \pi\left(\frac{l(l-1)}{2}, \frac{l(l+1)}{2}\right), \quad \text { or } \quad 8 \pi\left(\frac{l(l+1)}{2}, \frac{l(l-1)}{2}\right)
$$

for some integer $l>0$. Conversely, in [1] the authors have tried to construct blow-up solutions to (2) with Dirichlet boundary condition for $N=2$ and the following result was established: for $\varepsilon$ positive and small enough, there exist at least two pairs of solutions, which change sign exactly once, concentrate in the domain and whose nodal lines intersect the boundary. Later on, in [20] and [22] the authors dealt with the sinh-Poisson equation with homogenous Neumann boundary condition and showed a concentration phenomenon of solutions in the domain [20] and on the boundary [22] respectively.

Our motivation in (1) are two-folds. First, equation (1) is a natural generalization of (2) with zero Dirichlet boundary condition. One may expect similar results hold. (In fact, there a striking difference, as we will show below.) Secondly, equation (1) is a special case of equation (2) in higher-dimension $(N \geq 3)$. In fact, when we work with the standard $\mathrm{N}$ dimensional torus having axial symmetry, we can find that problem (2) is reduced to (1): let the torus be $T=\left\{x=\left(x_{i}\right) \in \mathbb{R}^{N}:\left(\sqrt{x_{1}^{2}+\cdots+x_{N-1}^{2}}-1\right)^{2}+x_{N}^{2} \leq r_{0}^{2}\right\}$ with $r_{0}<1$. If we look for solutions in the form $u(x)=u(r, s)$ with $r=\sqrt{x_{1}^{2}+\cdots+x_{N-1}^{2}}$ and $s=x_{N}$ for problem (2), a direct calculus shows that the problem is transformed to

$\operatorname{div}(r \nabla u)+2 \varepsilon^{2} r \sinh u=0$ in $\Omega_{T}=\left\{(r, s):(r-1)^{2}+s^{2}<r_{0}^{2}\right\}, \quad u=0$ on $\partial \Omega_{T}$ which is just the equation (1) with $a(r, s)=r$.

In this paper, we want to show the existence of solutions to (1), which blow up positively and negatively at a same point. This is quite different with results mentioned above and suggests that the dimension of the domain plays an important role in analysis of asymptotical behavior of solutions to (2).

To state our results, we need to introduce some notations. Let

$$
\Delta_{a} u=\frac{1}{a(x)} \operatorname{div}(a(x) \nabla u)=\Delta u+\nabla \log a \nabla u
$$


and $G(x, y)$ be the Green's function satisfying

$$
\Delta_{a} G(x, y)+8 \pi \delta_{y}=0 \text { in } \Omega, \quad G(x, y)=0 \text { on } \partial \Omega .
$$

We decompose $G(x, y)$ as

$$
G(x, y)=-4 \log |x-y|+H(x, y) .
$$

Let $m$ is an any positive integer, $b_{j}= \pm 1$ for any $j=1, \ldots, m$ and set

$$
\begin{aligned}
\mathcal{F}\left(\xi_{1}, \ldots, \xi_{m}\right)= & -16 \pi \sum_{j=1}^{m} a\left(\xi_{j}\right) \log \varepsilon+8 \pi(\log 8-2) \sum_{j=1}^{m} a\left(\xi_{j}\right) \\
& -4 \pi\left[\sum_{j=1}^{m} a\left(\xi_{j}\right) H\left(\xi_{j}, \xi_{j}\right)+\sum_{i \neq j} b_{i} b_{j} a\left(\xi_{j}\right) G\left(\xi_{i}, \xi_{j}\right)\right] .
\end{aligned}
$$

Now, our first result for the special case $m=2$ and $b_{1} b_{2}=-1$ can be stated as follows.

Theorem 1.1. Let $\bar{x} \in \Omega$ be a strict local minimum point of $a(x)$, i.e. there exists an neighborhood $B_{\delta}(\bar{x})$ such that $\delta>0, a(x)>a(\bar{x})$, for all $x \in B_{\delta}(\bar{x}) \backslash\{\bar{x}\}$. Then, problem (1) has a family of solutions $u_{\varepsilon}$ such that as $\varepsilon \rightarrow 0$,

$$
2 \varepsilon^{2} a(x) \sinh u_{\varepsilon} \rightarrow 0
$$

in the sense of measure in $\bar{\Omega}$. More precisely, we have

$$
u_{\varepsilon}(x)=\sum_{j=1}^{2} b_{j}\left[\log \frac{1}{\left(\varepsilon^{2} \mu_{j}^{2}+\left|x-\xi_{j}^{\varepsilon}\right|^{2}\right)^{2}}+H\left(x, \xi_{j}^{\varepsilon}\right)\right]+o(1)
$$

where $b_{j}= \pm 1(j=1,2)$ satisfying $b_{1} b_{2}=-1$ and $\mu_{j}$ satisfies

$$
|\log \varepsilon|^{-C} \leq \mu_{j} \leq|\log \varepsilon|^{C}
$$

and $\left(\xi_{1}^{\varepsilon}, \xi_{2}^{\varepsilon}\right)$ satisfies

$$
\xi_{j}^{\varepsilon} \rightarrow \bar{x} \text { for } j=1,2 \text { and }\left|\xi_{1}^{\varepsilon}-\xi_{2}^{\varepsilon}\right|>|\log \varepsilon|^{-\frac{5}{2}} .
$$

The theorem presents the existence of sign changing solutions for (1) with both positive and negative bubbles near a same point $\bar{x}$ for the Dirichlet Problem (1). It is quite surprising that such accumulation of bubbles can occur for anisotropic sinh-Poisson equation with Dirichlet boundary condition. Our result is different from that in $[9,15]$. In $[15]$ the authors showed that if the solutions concentrate positively and negatively at a same point, then the relation (3) must hold; And in [9] for a Neumann problem, a solution was constructed by superposing a positive and three negative bubbles centered near the origin.

For the general case that $m$ is an any positive integer, it is difficult for us to find the critical points of $\mathcal{F}$. So let us recall the following notion of stability critical points introduced in [8]:

Definition 1.2. We say that $\xi^{*}$ is a $C^{0}$-stable critical point of $F: \mathcal{O} \rightarrow \mathbb{R}$ if for any sequence of functions $F_{n}: \mathcal{O} \rightarrow \mathbb{R}$ such that $F_{n} \rightarrow F$ uniformly on compact sets of $\mathcal{O}$, we have that $F_{n}$ has a critical point $\xi_{n}$ such that $F_{n}\left(\xi_{n}\right) \rightarrow F\left(\xi^{*}\right)$. 
In particular, if $\xi$ is a strict local minimum/maximum point of $F$, then $\xi$ is a $C^{0}$-stable critical point. Next, we have the following result.

Theorem 1.3. Let $\bar{x} \in \Omega$ be a critical point of $a(x)$, for any positive integer $m$ and $b_{j}= \pm 1(j=1, \ldots, m)$, assume that $\left(\xi_{1}^{*}, \ldots, \xi_{m}^{*}\right) \in \mathcal{M}(\mathcal{M}$ defined by (15)) is a $C^{0}$-stable critical point for the function $\mathcal{F}$ defined in (6). Then, problem (1) has a family of solutions $u_{\varepsilon}$ such that as $\varepsilon \rightarrow 0$,

$$
2 \varepsilon^{2} \int_{\Omega} a(x) \sinh u \rightarrow 8 \pi \sum_{j=1}^{m} b_{j} \delta_{\bar{x}} .
$$

More precisely, we have

$$
u_{\varepsilon}(x)=\sum_{j=1}^{m} b_{j}\left[\log \frac{1}{\left(\varepsilon^{2} \mu_{j}^{2}+\left|x-\xi_{j}^{\varepsilon}\right|^{2}\right)^{2}}+H\left(x, \xi_{j}^{\varepsilon}\right)\right]+o(1)
$$

where $\mu_{j}$ satisfies

$$
|\log \varepsilon|^{-C} \leq \mu_{j} \leq|\log \varepsilon|^{C}
$$

and $\left(\xi_{1}^{\varepsilon}, \ldots, \xi_{m}^{\varepsilon}\right)$ satisfies

$$
\xi_{j}^{\varepsilon} \rightarrow \bar{x} \text { for all } j \text { and }\left|\xi_{i}^{\varepsilon}-\xi_{j}^{\varepsilon}\right|>|\log \varepsilon|^{-\frac{m^{2}+1}{2}}, \forall i \neq j .
$$

Theorems 1.1 and 1.3 are proved via the so-called "localized energy method" - a combination of Liapunov-Schmidt reduction method and variational techniques. Namely, we first use Liapunov-Schmidt reduction method to reduce the problem to a finite dimensional one, with some reduced energy. Then, the solutions in Theorems 1.3 turn out to be generated by critical points of the reduced energy functionals. Such an idea has been used in many other papers. See for instance $[2,4-7,11-13,18]$ and references therein. Here we follow those of [21] and introduce an appropriate functional setting, since the distance between the bubbles is small (see Theorem 1.3), but the large extent of $\mu_{j}$ bring us some difficulty in the estimates in Sect. 3 .

Throughout the paper, the symbol $C$ denotes always a positive constant independent of $\varepsilon$, it could be changed from one line to another.

\section{Ansatz for the solution}

The purpose of this section is to provide an ansatz for solutions of problem (1) and give some basic estimates for the "error term" in the corresponding scaling problem.

First, we point out that the function $H(x, y)$ defined by (5) plays an important role in our context. Especially, the argument that the function $x \mapsto H(x, x) \in C^{1}(\partial \Omega)$ is crucial for us to get the $C^{1}$ dependence of solution for the linearized problem, for the nonlinear problem and also for the variational reduction. So we recall the following expansion of $x \mapsto H(x, y)$ proved in [21]. 
Lemma 2.1. Let $H_{y}(x)=H(x, y), \forall y \in \Omega$. Then $y \mapsto H_{y} \in C\left(\Omega, C^{\gamma}(\bar{\Omega})\right)$ for any $\gamma \in(0,1)$. Let $H_{D}$ be the regular part of the standard Green's function of $-\Delta$ with zero Dirichlet boundary condition, then we have

$$
H(x, y)=8 \pi H_{D}(x, y)+\nabla \log a(y) \cdot \nabla_{x}\left(|x-y|^{2} \log |x-y|\right)+H_{1}(x, y)
$$

where $x \mapsto H_{1}(x, y) \in C^{1, \gamma}(\bar{\Omega})$ for all $\gamma \in(0,1)$. Furthermore, the function $(x, y) \mapsto H_{1}(x, y) \in C^{1}(\Omega \times \Omega)$, in particular the corresponding Robin function $x \mapsto H(x, x) \in C^{1}(\Omega)$.

Given now $\xi_{j} \in \Omega, \mu_{j}>0$, we define

$$
u_{j}(x)=\log \frac{8 \mu_{j}^{2}}{\left(\varepsilon^{2} \mu_{j}^{2}+\left|x-\xi_{j}\right|^{2}\right)^{2}},
$$

the choice of $\xi_{j}$ and $\mu_{j}$ will be made later on. Let $m \geq 1$ be an integer, then the configuration space for $\left(\xi_{1}, \ldots, \xi_{m}\right)$ we choose is the following

$$
\mathcal{M}:=\left\{\xi=\left(\xi_{1}, \ldots, \xi_{m}\right) \in B_{\delta}(\bar{x}) \times \cdots \times B_{\delta}(\bar{x})\left|\min _{i \neq j}\right| \xi_{i}-\left.\xi_{j}|\geq| \log \varepsilon\right|^{-M}\right\},
$$

where $M$ is given by

$$
M=\frac{m^{2}+1}{2} .
$$

Note that by the choice of $\xi_{j}$, we have if $\left(\xi_{1}, \ldots, \xi_{m}\right) \in \mathcal{M}$,

$$
|\log | \xi_{i}-\xi_{j}|| \leq C \log |\log \varepsilon|, \quad \forall i \neq j .
$$

We make the following ansatz for an approximate solution to (1)

$$
U(x)=\sum_{j=1}^{m} b_{j}\left[u_{j}(x)+H_{j}^{\varepsilon}(x)\right],
$$

where $H_{j}^{\varepsilon}$ is a correction term defined as the solution of

$$
\Delta_{a} H_{j}^{\varepsilon}+\nabla \log a(x) \nabla u_{j}=0 \quad \text { in } \Omega, \quad H_{j}^{\varepsilon}=-u_{j} \quad \text { on } \partial \Omega .
$$

By the same proof as for Lemma 2.4 in [21], we can obtain the following result.

Lemma 2.2. For any $0<\alpha<1$,

$$
H_{j}^{\varepsilon}(x)=H\left(x, \xi_{j}\right)-\log \left(8 \mu_{j}^{2}\right)+O\left(\varepsilon^{\alpha}\right)
$$

uniformly in $\bar{\Omega}$, where $H$ is the regular part of Green's function defined by (5).

It will be convenient to work with the scaling of $u$ given by $v(y)=u(\varepsilon y)$. If $u$ is a solution of (1) then $v$ satisfies

$$
\Delta_{a(\varepsilon y)} v+2 \varepsilon^{4} \sinh v=0 \text { in } \Omega_{\varepsilon}, \quad v=0 \text { on } \partial \Omega_{\varepsilon},
$$

where $\Omega_{\varepsilon}=\Omega / \varepsilon$. We will seek a solution $v$ of (21) of the form $v=V+\phi$ where

$$
V(y)=U(\varepsilon y)=\sum_{j=1}^{m} b_{j}\left[u_{j}(\varepsilon y)+H_{j}^{\varepsilon}(\varepsilon y)\right]
$$


and $U$ is defined by (18). Problem (21) can be stated as to find $\phi$ a solution to

$$
\Delta_{a(\varepsilon y)} \phi+W \phi+N(\phi)+R=0 \text { in } \Omega_{\varepsilon}, \quad \phi=0 \text { on } \partial \Omega_{\varepsilon},
$$

where

$$
W=2 \varepsilon^{4} \cosh V,
$$

$N$ stands for the "nonlinear term"

$$
N(\phi)=2 \varepsilon^{4}[\sinh (V+\phi)-\phi \cosh V-\sinh V]
$$

and $R$ is the "error term"

$$
R=\Delta_{a(\varepsilon y)} V+2 \varepsilon^{4} \sinh V .
$$

At this point it is convenient to make a choice of the parameters $\mu_{j}$, the objective being to make the error term small. We choose $\mu_{j}$ such that

$$
\log \left(8 \mu_{j}^{2}\right)=H\left(\xi_{j}, \xi_{j}\right)+\sum_{i \neq j} b_{i} b_{j} G\left(\xi_{i}, \xi_{j}\right), \quad \forall j .
$$

Since $\xi_{j} \rightarrow \bar{x}$ implies that $\lim G\left(\xi_{i}, \xi_{j}\right)=\infty$ and the fact

$$
0<G\left(\xi_{i}, \xi_{j}\right) \leq-4 \log \left|\xi_{i}-\xi_{j}\right|+O(1) \leq C \log |\log \varepsilon|,
$$

for some fixed positive number $C$ we derive that

$$
\frac{1}{C|\log \varepsilon|^{2 M}} \leq \mu_{j} \leq C|\log \varepsilon|^{2 M} .
$$

By the Appendix A, we obtain that the following behavior for $R$ holds: for any $0<\alpha<1$, there exists $C$ independent of $\varepsilon$ such that

$$
|R(y)| \leq C \varepsilon^{\alpha} \sum_{j=1}^{m} \frac{1}{\mu_{j}^{2}\left(1+\mu_{j}^{-3}\left|y-\xi_{j}^{\prime}\right|^{3}\right)}, \quad \forall y \in \Omega_{\varepsilon}
$$

and for $W=2 \varepsilon^{4} \cosh V$,

$$
W(y)= \begin{cases}\frac{8 \mu_{j}^{2}}{\left(\mu_{j}^{2}+\left|y-\xi_{j}^{\prime}\right|^{2}\right)^{2}}\left(1+\theta_{j}(y)\right)+O\left(\varepsilon^{4-\alpha}\right) & \text { if }\left|y-\xi_{j}^{\prime}\right| \leq \frac{1}{\varepsilon|\log \varepsilon|^{2 M}} \\ O\left(\varepsilon^{4-\alpha}\right) & \text { if }\left|y-\xi_{j}^{\prime}\right| \geq \frac{1}{\varepsilon|\log \varepsilon|^{2 M}}, \forall j\end{cases}
$$

with $\theta_{j}$ satisfying the estimate $\left|\theta_{j}(y)\right| \leq C_{\alpha}\left(\varepsilon^{\alpha}\left|y-\xi_{j}^{\prime}\right|^{\alpha}+\varepsilon^{\alpha}\right)$, where $\xi_{j}^{\prime}=$ $\xi_{j} / \varepsilon$.

\section{Linearized equations and nonlinear problem}

The first main result of this section is the solvability of the following linear problem: Given $h \in L^{\infty}\left(\Omega_{\varepsilon}\right)$, find $\phi, c_{11}, \ldots, c_{2 m}$ such that

$$
\left\{\begin{aligned}
-\Delta_{a(\varepsilon y)} \phi & =W \phi+h+\frac{1}{a(\varepsilon y)} \sum_{j=1}^{m} \sum_{i=1}^{2} c_{i j} \chi_{j} Z_{i j} & & \text { in } \Omega_{\varepsilon} \\
\phi & =0 & & \text { on } \partial \Omega_{\varepsilon} \\
\int_{\Omega_{\varepsilon}} \chi_{j} Z_{i j} \phi & =0, \quad \forall 1 \leq j \leq m, i=1,2 & &
\end{aligned}\right.
$$


where $W$ is a function satisfying (29), and $Z_{i j}, \chi_{j}$ are defined as follows. Let $z_{i j}$ be

$$
z_{0 j}=\frac{1}{\mu_{j}}-2 \frac{\mu_{j}}{\mu_{j}^{2}+|y|^{2}}, \quad z_{i j}=\frac{y_{i}}{\mu_{j}^{2}+|y|^{2}}, \quad 1 \leq i \leq 2, \quad 1 \leq j \leq m .
$$

It is well-known that any bounded solution to

$$
\Delta \phi+\frac{8}{\left(1+|z|^{2}\right)^{2}} \phi=0
$$

is a linear combination of $Z_{i}, i=0,1,2$ where $Z_{0}=\frac{1-|z|^{2}}{1+|z|^{2}}$ and $Z_{i}=\frac{z_{i}}{1+|z|^{2}}$ for $i=1,2$ (See lemma 2.1 of [3]).

Next we choose a large but fixed number $R_{0}$ and nonnegative smooth function $\chi: \mathbb{R} \rightarrow \mathbb{R}$ so that $\chi(r)=1$ for $r \leq R_{0}$ and $\chi(r)=0$ for $r \geq$ $R_{0}+1,0 \leq \chi \leq 1$. For $j=1, \ldots, m$, we define

$$
\chi_{j}(y)=\chi\left(\frac{\left|y-\xi_{j}^{\prime}\right|}{\mu_{j}}\right), \quad Z_{i j}(y)=z_{i j}\left(y-\xi_{j}^{\prime}\right), \quad i=0,1,2 ; j=1, \ldots, m .
$$

It is important to note that

$$
\begin{aligned}
& \Delta_{a(\varepsilon y)} Z_{0 j}+\log \frac{8 \mu_{j}^{2}}{\left(\mu_{j}^{2}+\left|y-\xi_{j}^{\prime}\right|^{2}\right)^{2}} Z_{0 j}=\varepsilon \nabla \log a(\varepsilon y) \nabla Z_{0 j} \\
& =O\left(\varepsilon \mu_{j}^{-2}\left[1+\frac{\left|y-\xi_{j}^{\prime}\right|}{\mu_{j}}\right]^{-3}\right) .
\end{aligned}
$$

All functions above depend on $\varepsilon$ but we omit this dependence in the notation as for $\xi_{j}^{\prime}$.

Equation (30) will be solved for $h \in L^{\infty}\left(\Omega_{\varepsilon}\right)$, but we will be able to estimate the size of the solution in terms of the following norm

$$
\|h\|_{*}=\sup _{y \in \Omega_{\varepsilon}} \frac{|h(y)|}{\varepsilon^{2}+\sum_{j=1}^{m} \mu_{j}^{-2}\left(1+\frac{\left|y-\xi_{j}^{\prime}\right|}{\mu_{j}}\right)^{-3}} .
$$

Proposition 3.1. Let $m$ be a positive integer. Then there exist $\varepsilon_{0}>0, C>0$ such that for any $0<\varepsilon<\varepsilon_{0}$, any family of points $\xi=\left(\xi_{1}, \ldots, \xi_{m}\right) \in \mathcal{M}$ and any $h \in L^{\infty}\left(\Omega_{\varepsilon}\right)$, there is a unique solution $\phi \in L^{\infty}\left(\Omega_{\varepsilon}\right), c_{i j} \in \mathbb{R}$ to (30). Moreover

$$
\|\phi\|_{L^{\infty}\left(\Omega_{\varepsilon}\right)} \leq C|\log \varepsilon|\|h\|_{*} .
$$

Proof. We carry the proof out in following steps.

Step 1. Solve a linearized problem with orthogonality conditions to all $Z_{i j}, 0 \leq i \leq 2,1 \leq j \leq m$. First, we consider the following linearized problem.

Lemma 3.2. There are $R_{0}>0$ and $\varepsilon_{0}>0$ so that for $0<\varepsilon<\varepsilon_{0}$ and any solution $\phi$ of

$$
\left\{\begin{aligned}
-\Delta_{a(\varepsilon y)} \phi & =W \phi+h & & \text { in } \Omega_{\varepsilon} \\
\phi & =0 & & \text { on } \partial \Omega_{\varepsilon} \\
\int_{\Omega_{\varepsilon}} \chi_{j} Z_{i j} \phi & =0 & & \forall j=1, \ldots, m ; i=0,1,2,
\end{aligned}\right.
$$


we have

$$
\|\phi\|_{L^{\infty}\left(\Omega_{\varepsilon}\right)} \leq C\|h\|_{*}
$$

where $C$ is independent of $\varepsilon \in\left(0, \varepsilon_{0}\right)$.

For the proof of this lemma, one can find in the section 3 in [21].

Step 2. Establish an a-priori estimate for solutions to (34) that satisfy orthogonality conditions with respect to $Z_{i j}$ for $i=1,2$ only. More precisely, we have the following result.

Lemma 3.3. For $\varepsilon>0$ sufficiently small, if $\phi$ solves

$$
-\Delta_{a(\varepsilon y)} \phi-W \phi=h \quad \text { in } \Omega_{\varepsilon}, \quad \phi=0 \quad \text { on } \partial \Omega_{\varepsilon}
$$

and satisfies

$$
\int_{\Omega_{\varepsilon}} Z_{i j} \chi_{j} \phi=0 \quad \forall j=1, \ldots, m ; i=1,2,
$$

there exists a constant $C$ which is independent of $\varepsilon$ such that

$$
\|\phi\|_{L^{\infty}\left(\Omega_{\varepsilon}\right)} \leq C|\log \varepsilon|\|h\|_{*} .
$$

Proof. Let $\phi$ satisfy (35) and (36). We will modify $\phi$ to satisfy all orthogonality relations in (34) and for this purpose we consider modifications with compact support of the functions $Z_{0 j}$. Let $R>R_{0}+1$ be large and fixed. Let

$$
a_{0 j}=\frac{1}{\mu_{j}\left[-4 \log \left(\varepsilon \mu_{j} R\right)+H\left(\xi_{j}, \xi_{j}\right)\right]} .
$$

Let $\eta_{1}$ be radial smooth cut-off function on $\mathbb{R}^{2}$ so that

$$
0 \leq \eta_{1} \leq 1, \quad\left|\nabla \eta_{1}\right| \leq C \text { in } \mathbb{R}^{2} ; \quad \eta_{1} \equiv 1 \text { in } B_{R}(0), \quad \eta_{1} \equiv 0 \text { in } \mathbb{R}^{2} \backslash B_{R+1}(0)
$$

and $\eta_{2}$ be radial smooth cut-off function on $\mathbb{R}^{2}$ so that

$$
0 \leq \eta_{2} \leq 1, \quad\left|\nabla \eta_{2}\right| \leq C \text { in } \mathbb{R}^{2} ; \quad \eta_{2} \equiv 1 \text { in } B_{1}(0), \quad \eta_{2} \equiv 0 \text { in } \mathbb{R}^{2} \backslash B_{2}(0) .
$$

Without loss of generality, we assume that $\delta<1$ and $B(\bar{x}, 2+\delta) \subset \Omega$. Let

$$
\eta_{1 j}(y)=\eta_{1}\left(\frac{\left|y-\xi_{j}^{\prime}\right|}{\mu_{j}}\right), \quad \eta_{2 j}(y)=\eta_{2}\left(4 \varepsilon\left|y-\xi_{j}^{\prime}\right|\right)
$$

and also

$$
\widehat{Z}_{0 j}(y)=Z_{0 j}(y)-\frac{1}{\mu_{j}}+a_{0 j} G\left(\varepsilon y, \xi_{j}\right) .
$$

Now define $\widetilde{Z}_{0 j}=\eta_{1 j} Z_{0 j}+\left(1-\eta_{1 j}\right) \eta_{2 j} \widehat{Z}_{0 j}$. Given $\phi$ satisfying (35) and (36), let

$$
\widetilde{\phi}=\phi+\sum_{j=1}^{m} d_{j} \widetilde{Z}_{0 j}+\sum_{i=1}^{2} \sum_{j=1}^{m} e_{i j} Z_{i j} \chi_{j} .
$$

We adjust $\widetilde{\phi}$ to satisfy the orthogonality condition:

$$
\int_{\Omega_{\varepsilon}} \chi_{j} Z_{i j} \widetilde{\phi}=0, \quad \text { for all } 0 \leq i \leq 2,1 \leq j \leq m .
$$


We prove the existence of $d_{j}$ and $e_{i j}$. First, take $\chi_{j} Z_{i j}$ and $\chi_{l} Z_{0 l}$ as test functions for (41), we obtain that $e_{i j}$ satisfies

$$
e_{i j}=-\frac{\int_{\Omega_{\varepsilon}} \sum_{l \neq j} d_{l} \widetilde{Z}_{0 l} Z_{i j} \chi_{j}}{\int_{\Omega_{\varepsilon}} Z_{i j}^{2} \chi_{j}^{2}}
$$

and $d_{j}$ satisfy the system

$$
\sum_{j} d_{j} \int_{\Omega_{\varepsilon}} \widetilde{Z}_{0 j} Z_{0 l} \chi_{l}=-\int_{\Omega_{\varepsilon}} \phi Z_{0 l} \chi_{l}, \quad \text { for } \quad l=1, \ldots, m .
$$

Note that

$$
\int_{\Omega_{\varepsilon}} \widetilde{Z}_{0 l} Z_{0 l} \chi_{l}=\int_{B_{\mu_{l} R}\left(\xi_{l}^{\prime}\right)} Z_{0 l}^{2} \chi_{l}=C, \quad \forall l
$$

and

$$
\int_{\Omega_{\varepsilon}} \widetilde{Z}_{0 j} Z_{0 l} \chi_{l}=\int_{B_{\mu_{l} R}\left(\xi_{l}^{\prime}\right)} \widehat{Z}_{0 j} Z_{0 l} \chi_{l}=O\left(\frac{\mu_{l} \log |\log \varepsilon|}{\mu_{j}|\log \varepsilon|}\right), \quad \forall j \neq l,
$$

where we have used $\left|\xi_{l}^{\prime}-\xi_{j}^{\prime}\right| \geq \varepsilon^{-1}|\log \varepsilon|^{-M}$ and $|\log \varepsilon|^{-C} \leq \mu_{j} \leq|\log \varepsilon|^{C}$ $(j=1, \ldots, m)$. Thus we deduce that the coefficient matrix of system (43) is diagonally dominated, hence $\left(d_{1}, \ldots, d_{m}\right)$ is well defined.

Estimate (37) is a direct consequence of the following two claims:

Claim 1. Let $L=-\Delta_{a(\varepsilon y)}-W$, then

$$
\left\|L\left(Z_{i j} \chi_{j}\right)\right\|_{*} \leq \frac{C}{\mu_{j}}, \quad \forall i=1,2 ; j=1, \ldots, m,
$$

and

$$
\left\|L \widetilde{Z}_{0 j}\right\|_{*} \leq C \frac{\log |\log \varepsilon|}{\mu_{j}|\log \varepsilon|}, \quad \forall j=1, \ldots, m
$$

\section{Claim 2.}

$$
\left|d_{j}\right| \leq C \mu_{j}|\log \varepsilon|\|h\|_{*}, \quad\left|e_{i j}\right| \leq C \mu_{j}|\log \varepsilon|\|h\|_{*} \quad \forall i=1,2 ; j=1, \ldots, m .
$$

In fact, we have that

$$
L(\widetilde{\phi})=h+\sum_{j=1}^{m} d_{j} L\left(\widetilde{Z}_{0 j}\right)+\sum_{i=1}^{2} \sum_{j=1}^{m} e_{i j} L\left(Z_{i j} \chi_{j}\right) \quad \text { in } \Omega_{\varepsilon} .
$$

Thus by Lemma 3.2, we have

$$
\begin{aligned}
\|\widetilde{\phi}\|_{L^{\infty}\left(\Omega_{\varepsilon}\right)} & \leq C\|h\|_{*}+C \sum_{j=1}^{m}\left|d_{j}\right|\left\|L\left(\widetilde{Z}_{0 j}\right)\right\|_{*}+C \sum_{i=1}^{2} \sum_{j=1}^{m}\left|e_{i j}\right|\left\|L\left(Z_{i j} \chi_{j}\right)\right\|_{*} \\
& \leq C|\log \varepsilon|\|h\|_{*} .
\end{aligned}
$$

Using (41), the estimate (37) then follows from (48), Claims 1 and 2.

We will prove Claim 1 in the following. As for the proof of Claim 2, one can do it following the calculations in section 3 in [21], here we omit the details. 
Proof of Claim 1. First, the domain $\Omega_{\varepsilon}$ splits into four subregion as follows:

$$
\begin{array}{ll}
\Omega_{1}=\left\{\left|y-\xi_{j}^{\prime}\right| \leq \mu_{j} R\right\}, & \Omega_{2}=\left\{\mu_{j} R \leq\left|y-\xi_{j}^{\prime}\right| \leq \mu_{j}(R+1)\right\} \\
\Omega_{3}=\left\{\mu_{j}(R+1) \leq\left|y-\xi_{j}^{\prime}\right| \leq \frac{1}{4 \varepsilon}\right\}, & \Omega_{4}=\left\{\frac{1}{4 \varepsilon} \leq\left|y-\xi_{j}^{\prime}\right| \leq \frac{1}{2 \varepsilon}\right\} .
\end{array}
$$

We will estimate the sizes of $\left\|L\left(Z_{i j} \chi_{j}\right)\right\|_{*}$ and $\left\|L \widetilde{Z}_{0 j}\right\|_{*}$ on these subregion.

On $\Omega_{1}$, by the definitions of $\chi_{j}$ and $Z_{i j}$ we have

$$
L\left(Z_{i j} \chi_{j}\right)=-\Delta_{a(\varepsilon y)} Z_{i j}-W Z_{i j}=-\varepsilon \nabla A(\varepsilon y) \nabla Z_{i j}=O\left(\varepsilon \mu_{j}^{-2}\right) .
$$

On $\Omega_{2}$, note that $\nabla \chi_{j}=O\left(\mu_{j}^{-1}\right), \Delta \chi_{j}=O\left(\mu_{j}^{-2}\right)$ and $\nabla Z_{i j}=O\left(\mu_{j}^{-2}\right)$, we get

$$
L\left(Z_{i j} \chi_{j}\right)=-\Delta_{a(\varepsilon y)}\left(Z_{i j} \chi_{j}\right)-W Z_{i j} \chi_{j}=O\left(\mu_{j}^{-3}\right) .
$$

Thus, (44) follows.

Now we estimate the size of $\left\|L \widetilde{Z}_{0 j}\right\|_{*}$. By (29) and the definitions of $Z_{0 j}$, we easily obtain that on $\Omega_{1}$

$$
\left(e^{v_{j}}-W\right) Z_{0 j}=O\left(\varepsilon^{\alpha} \mu_{j}^{-2}\left[1+\frac{\left|y-\xi_{j}^{\prime}\right|}{\mu_{j}}\right]^{-4}\right) \times O\left(\mu_{j}^{-1}\right),
$$

so, taking account of (32) we have

$$
\begin{aligned}
L \widetilde{Z}_{0 j} & =L Z_{0 j}=O\left(\varepsilon \mu_{j}^{-2}\left[1+\frac{\left|y-\xi_{j}^{\prime}\right|}{\mu_{j}}\right]^{-3}\right)+\left(e^{v_{j}}-W\right) Z_{0 j} \\
& =O\left(\varepsilon \mu_{j}^{-2}\left[1+\frac{\left|y-\xi_{j}^{\prime}\right|}{\mu_{j}}\right]^{-3}\right)+O\left(\left(\varepsilon^{\alpha} \mu_{j}^{-1}\right) \mu_{j}^{-2}\left[1+\frac{\left|y-\xi_{j}^{\prime}\right|}{\mu_{j}}\right]^{-4}\right) \\
& =O\left(\varepsilon^{\beta} \mu_{j}^{-2}\left[1+\frac{\left|y-\xi_{j}^{\prime}\right|}{\mu_{j}}\right]^{-3}\right),
\end{aligned}
$$

where $\beta \in(0,1)$ fixed.

On $\Omega_{2}$, a direct computation shows

$$
\begin{aligned}
L \widetilde{Z}_{0 j}= & L Z_{0 j}-\left(1-\eta_{1 j}\right) W\left(\widehat{Z}_{0 j}-Z_{0 j}\right)+2 \nabla \eta_{1 j} \nabla\left(\widehat{Z}_{0 j}-Z_{0 j}\right) \\
& +\left(\widehat{Z}_{0 j}-Z_{0 j}\right) \Delta_{a(\varepsilon y)} \eta_{1 j} .
\end{aligned}
$$

Note that on $\Omega_{2}$

$\widehat{Z}_{0 j}-Z_{0 j}=-\frac{1}{\mu_{j}}+a_{0 j} G\left(\varepsilon y, \xi_{j}\right)=a_{0 j}\left[4 \log \frac{\mu_{j} R}{\left|y-\xi_{j}^{\prime}\right|}+H\left(\varepsilon y, \xi_{j}\right)-H\left(\xi_{j}, \xi_{j}\right)\right]$,

and by (13) we find that on $\Omega_{2}$

$$
\widehat{Z}_{0 j}-Z_{0 j}=O\left(\mu_{j}^{-1}|\log \varepsilon|^{-1}\right), \quad \nabla\left(\widehat{Z}_{0 j}-Z_{0 j}\right)=O\left(\mu_{j}^{-2}|\log \varepsilon|^{-1}\right) .
$$

At the same time, observe that $\left|\nabla \eta_{1 j}\right|=O\left(\mu_{j}^{-1}\right), \Delta_{a(\varepsilon y)} \eta_{1 j}=O\left(\mu_{j}^{-2}\right)$, we deduce that

$$
\left\|L \widetilde{Z}_{0 j}\right\|_{L^{\infty}\left(\Omega_{2}\right)}=O\left(\mu_{j}^{-3}|\log \varepsilon|^{-1}\right) .
$$

On $\Omega_{3}$, we have

$$
L \widetilde{Z}_{0 j}=\left(-\Delta_{a(\varepsilon y)}-e^{v_{j}}\right) Z_{0 j}+\left(e^{v_{j}}-W\right) Z_{0 j}+W\left[\frac{1}{\mu_{j}}-a_{0 j} G\left(\varepsilon y, \xi_{j}\right)\right] \text {. }
$$


To estimate the last two terms in the above equation, we separate $\Omega_{3}$ into the following subregions:

$$
\begin{aligned}
& \Omega_{3 j}=\left\{\mu_{j}(R+1)<\left|y-\xi_{j}^{\prime}\right| \leq \frac{1}{\varepsilon|\log \varepsilon|^{2 M}}\right\}, \\
& \Omega_{3 l}=\left\{y \in \Omega_{3},\left|y-\xi_{l}^{\prime}\right| \leq \frac{1}{\varepsilon|\log \varepsilon|^{2 M}} \quad \text { if } l \neq j\right\}, \\
& \widetilde{\Omega}_{3}=\left\{y \in \Omega_{3},\left|y-\xi_{j}^{\prime}\right| \geq \frac{1}{\varepsilon|\log \varepsilon|^{2 M}}, \forall j\right\} .
\end{aligned}
$$

From (29) and the definition of $Z_{0 j}$, it follows easily that

$$
\begin{aligned}
& \left(e^{v_{j}}-W\right) Z_{0 j} \\
& \quad= \begin{cases}\frac{8 \mu_{j}}{\left(\mu_{j}^{2}+\left|y-\xi_{j}^{\prime}\right|^{2}\right)^{2}} \times O\left(\varepsilon^{\alpha}+\varepsilon^{\alpha}\left|y-\xi_{j}^{\prime}\right|^{\alpha}\right)+O\left(\varepsilon^{4-\alpha} \mu_{j}^{-1}\right) & \text { if } y \in \Omega_{3 j}, \\
O\left(\varepsilon^{4-\alpha} \mu_{j}^{-1}\right) & \text { if } y \in \widetilde{\Omega}_{3} .\end{cases}
\end{aligned}
$$

For $y \in \Omega_{3 j}$, we have

$$
\begin{aligned}
& W\left[\frac{1}{\mu_{j}}-a_{0 j} G\left(\varepsilon y, \xi_{j}\right)\right] \\
& =O\left(\frac{8 \mu_{j}^{2}}{\left(\mu_{j}^{2}+\left|y-\xi_{j}^{\prime}\right|^{2}\right)^{2}}+O\left(\varepsilon^{4-\alpha}\right)\right) \times a_{0 j} \times O\left(\log \frac{\left|y-\xi_{j}^{\prime}\right|}{\mu_{j} R}+\varepsilon^{\alpha}\left|y-\xi_{j}^{\prime}\right|^{\alpha}\right) \\
& =O\left(\mu_{j}^{-2}\left[1+\frac{\left|y-\xi_{j}^{\prime}\right|}{\mu_{j}}\right]^{-3} \frac{1}{\mu_{j}|\log \varepsilon|}\right) .
\end{aligned}
$$

For $y \in \widetilde{\Omega}_{3}, W\left[\mu_{j}^{-1}-a_{0 j} G\left(\varepsilon y, \xi_{j}\right)\right]=O\left(\varepsilon^{4-\alpha} \mu_{j}^{-1}\right)$. Therefore, we obtain

$$
\left|L \widetilde{Z}_{0 j}(y)\right|=O\left(\mu_{j}^{-2}\left[1+\frac{\left|y-\xi_{j}^{\prime}\right|}{\mu_{j}}\right]^{-3} \frac{1}{\mu_{j}|\log \varepsilon|}\right) \quad y \in \widetilde{\Omega}_{3} \cup \Omega_{3 j} .
$$

For $y \in \Omega_{3 l}(l \neq j)$, note that

$$
\begin{aligned}
e^{v_{j}} Z_{0 j} & =O\left(\varepsilon^{4-\alpha} \mu_{j}^{-1}\right), \quad W=O\left(\mu_{l}^{-2}\left[1+\frac{\left|y-\xi_{l}^{\prime}\right|}{\mu_{l}}\right]^{-4}\right) \text { and } \\
\left|\widehat{Z}_{0 j}\right| & =O\left(\frac{\log |\log \varepsilon|}{\mu_{j}|\log \varepsilon|}\right),
\end{aligned}
$$

we get

$$
e^{v_{j}} Z_{0 j}-W \widehat{Z}_{0 j}=O\left(\mu_{l}^{-2}\left[1+\frac{\left|y-\xi_{l}^{\prime}\right|}{\mu_{l}}\right]^{-3} \frac{\log |\log \varepsilon|}{\mu_{j}|\log \varepsilon|}\right) \quad y \in \Omega_{3 l}(l \neq j) .
$$

thus

$$
\left|L \widetilde{Z}_{0 j}(y)\right|=O\left(\frac{\log |\log \varepsilon|}{\mu_{j}|\log \varepsilon|}\right) \times \sum_{l=1}^{m} \mu_{l}^{-2}\left(1+\frac{\left|y-\xi_{l}^{\prime}\right|}{\mu_{l}}\right)^{-3}, \quad \forall y \in \Omega_{3} .
$$

On $\Omega_{4}$, we have

$$
\begin{aligned}
L \widetilde{Z}_{0 j}= & \eta_{2 j}\left[-\Delta_{a(\varepsilon y)} Z_{0 j}-e^{v_{j}} Z_{0 j}\right]-2 \nabla \widehat{Z}_{0 j} \nabla \eta_{2 j}-\widehat{Z}_{0 j} \Delta_{a(\varepsilon y)} \eta_{2 j} \\
& -\eta_{2 j}\left(W \widehat{Z}_{0 j}-e^{v_{j}} Z_{0 j}\right) .
\end{aligned}
$$


Since $\left|\nabla \eta_{2 j}\right|=O(\varepsilon),\left|\Delta_{a(\varepsilon y)} \eta_{2 j}\right|=O\left(\varepsilon^{2}\right),\left|\nabla Z_{0 j}\right|=O\left(\mu_{j}^{-2}\left[1+\frac{\left|y-\xi_{j}^{\prime}\right|}{\mu_{j}}\right]^{-3}\right)$ and $\left|\widehat{Z}_{0 j}\right|=O\left(\frac{1}{\mu_{j}|\log \varepsilon|}\right)$, we get

$$
\left\|L \widetilde{Z}_{0 j}\right\|_{L^{\infty}\left(\Omega_{4}\right)}=O\left(\frac{\varepsilon^{2}}{\mu_{j}|\log \varepsilon|}\right) .
$$

Combining (49), (51), (55) and (56), we deduce that (45) holds.

Step 3. Proof of Proposition 3.1.

First we prove that for any $\phi, c_{i j}$ solution to (30) the bound

$$
\|\phi\|_{L^{\infty}\left(\Omega_{\varepsilon}\right)} \leq C|\log \varepsilon|\|h\|_{*}
$$

holds. In fact, the previous lemma yields

$$
\|\phi\|_{L^{\infty}\left(\Omega_{\varepsilon}\right)} \leq C|\log \varepsilon|\left(\|h\|_{*}+\sum_{j=1}^{m} \sum_{i=1}^{2}\left|c_{i j}\right| \mu_{j}\right) .
$$

So it suffices to estimate the values of the constants $c_{i j}$. To this end, we multiply (30) by $a(\varepsilon y) \widetilde{Z}_{i j}$ with $\widetilde{Z}_{i j}=Z_{i j} \eta_{2 j}, \eta_{2 j}$ given by (39), and integrate to find

$$
\int_{\Omega_{\varepsilon}} a(\varepsilon y) \widetilde{Z}_{i j} L \phi=\int_{\Omega_{\varepsilon}} a(\varepsilon y) h \widetilde{Z}_{i j}+\sum_{k=1}^{2} \sum_{l=1}^{m} c_{k l} \int_{\Omega_{\varepsilon}} \widetilde{Z}_{i j} Z_{k l} \chi_{l} .
$$

The computations in section 3 in [21] show that $\left\|L \widetilde{Z}_{i j}\right\|_{*} \leq C \varepsilon^{1 / 3}$ for any $i=1,2$ and $j=1, \ldots, m$. Hence

$$
\int_{\Omega_{\varepsilon}} a(\varepsilon y) \widetilde{Z}_{i j} L \phi=\int_{\Omega_{\varepsilon}} a(\varepsilon y) \phi L \widetilde{Z}_{i j}=O\left(\varepsilon^{1 / 3}\|\phi\|_{\infty}\right) .
$$

Moreover

$$
\int_{\Omega_{\varepsilon}} a(\varepsilon y) h \widetilde{Z}_{i j}=O\left(\mu_{j}^{-1}\|h\|_{*}\right) .
$$

Now we need just to estimate the coefficient matrix for $c_{i j}$. By definition,

$$
\int_{\Omega_{\varepsilon}} \widetilde{Z}_{i l} Z_{k l} \chi_{l}=\int_{\Omega_{\varepsilon}} Z_{i l} Z_{k l} \chi_{l}=C \delta_{i k}
$$

and if $j \neq l$,

$$
\int_{\Omega_{\varepsilon}} \widetilde{Z}_{i j} Z_{k l} \chi_{l}=\int_{\Omega_{\varepsilon}} Z_{i j} Z_{k l} \chi_{l}=O\left(\varepsilon|\log \varepsilon|^{M} \mu_{l}\right),
$$

it is clear that the coefficient matrix is diagonally dominated and the inverse is uniformly bounded for small $\varepsilon$. Substituting (61) and (60) into (59), we obtain the estimate $\sup \left|c_{i j}\right| \leq C\left(\mu_{j}^{-1}\|h\|_{*}+\varepsilon^{1 / 3}\|\phi\|_{\infty}\right)$ and thus (57).

Now consider the Hilbert space

$$
H=\left\{\phi \in H_{0}^{1}\left(\Omega_{\varepsilon}\right): \int_{\Omega_{\varepsilon}} \chi_{j} Z_{i j} \phi=0 \quad \forall j=1, \ldots, m ; i=1,2\right\}
$$


with the norm $\|\phi\|_{H_{0}^{1}}=\|\nabla \phi\|_{L^{2}\left(\Omega_{\varepsilon}\right)}$. Equation (30) is equivalent to find $\phi \in H$ such that

$$
\int_{\Omega_{\varepsilon}} a(\varepsilon y) \nabla \phi \nabla \psi-a(\varepsilon y) W \phi \psi=\int_{\Omega_{\varepsilon}} a(\varepsilon y) h \psi, \quad \forall \psi \in H .
$$

By Fredholm's alternative this is equivalent to the uniqueness of solutions to this problem, which is guaranteed by (57).

The result of Proposition 3.1 implies that the unique solution $\phi=T(h)$ of (30) defines a continuous linear map from the Banach space $\mathcal{C}_{*}$ of all functions $h$ in $L^{\infty}$ for which $\|h\|_{*}<\infty$, into $L^{\infty}$. We need to understand the differentiability of the operator $T$ with respect to the variable $\xi^{\prime}$. Fix $h \in \mathcal{C}_{*}$ and let $\phi=T(h)$. We can compute the derivatives of $\phi$ with respect to $\xi^{\prime}$ and obtain their estimates as follows

$$
\left\|\partial_{\xi^{\prime}} T(h)\right\|_{\infty} \leq C|\log \varepsilon|^{2 M+3}\|h\|_{*}, \quad \text { for all } k=1, \ldots, m ; l=1,2 .
$$

Sketch of proof. The proof is similar to that in [5], the most delicate point is to estimate $\left\|\partial_{\xi^{\prime}} W\right\|_{*}$. Since $W=2 \varepsilon^{4} \cosh V$, we need just to estimate $\left\|\partial_{\xi^{\prime}} V\right\|_{\infty}$.

For that, we consider first $\mu_{j}$. Thanks to (26) and Lemma 2.1, we prove easily that $\left|\partial_{\xi} \mu_{j}\right|=O\left(\mu_{j}|\log \varepsilon|^{M}\right)$ over $\mathcal{M}$. This will lead to $\left\|\partial_{\xi} u_{j}\right\|_{\infty}=$ $O\left(\varepsilon^{-1} \mu_{j}^{-1}\right)$. Now using the equation for the ansatz $U$ and by maximum principle, we get

$$
\left\|\partial_{\xi} U\right\|_{\infty} \leq\left\|\partial_{\xi} u_{j}\right\|_{\infty}\|U\|_{\infty}=O\left(\frac{|\log \varepsilon|^{2 M+1}}{\varepsilon}\right) .
$$

After the scaling, we obtain finally $\left\|\partial_{\xi^{\prime}} V\right\|_{\infty}=O\left(|\log \varepsilon|^{2 M+1}\right)$. It follows from (76) that $\left\|2 \varepsilon^{4} \sinh V\right\|_{*} \leq C$, hence (62) holds.

Now we show the nonlinear equation associated to $V$

$$
\left\{\begin{aligned}
-\Delta_{a(\varepsilon y)} \phi-W \phi & =R+N(\phi)+\frac{1}{a(\varepsilon y)} \sum_{i, j} c_{i j} \chi_{j} Z_{i j} & & \text { in } \Omega_{\varepsilon} \\
\phi & =0 & & \text { on } \partial \Omega_{\varepsilon} \\
\int_{\Omega_{\varepsilon}} \chi_{j} Z_{i j} \phi & =0, \quad \forall j=1, \ldots, m, i=1,2 & &
\end{aligned}\right.
$$

where $W$ is as in (29) and $N, R$ are defined in (24) and (25) respectively. We have the following result.

Lemma 3.4. Let $\alpha \in(0,1)$. Then there exist $\varepsilon_{0}>0, C>0$ such that for any $0<\varepsilon<\varepsilon_{0}$ and any $\xi=\left(\xi_{1}, \ldots, \xi_{m}\right) \in \mathcal{M}$ the problem (63) admits a unique solution $\phi, c_{i j}$ such that

$$
\|\phi\|_{L^{\infty}\left(\Omega_{\varepsilon}\right)} \leq C \varepsilon^{\alpha}|\log \varepsilon| .
$$

Furthermore, the function $\xi^{\prime} \mapsto \phi\left(\xi^{\prime}\right)$ is $C^{1}$ and

$$
\left\|\nabla_{\xi^{\prime}} \phi\right\|_{L^{\infty}\left(\Omega_{\varepsilon}\right)} \leq C \varepsilon^{\alpha}|\log \varepsilon|^{2 M+3} \text {. }
$$

Proof. The proof of this lemma can be done along the lines of those of lemma 4.1 of [5]. Here we should point out that since $\|W\|_{*} \leq O(1),\|R\|_{*} \leq$ $O\left(\varepsilon^{\alpha}\right)$ and $\|N(\phi)\|_{*} \leq C\|\phi\|_{L^{\infty}\left(\Omega_{\varepsilon}\right)}^{2}$, so we can apply the Contraction Mapping Theorem to the nonlinear problem in the region

$$
\mathcal{S}=\left\{\phi \in C\left(\bar{\Omega}_{\varepsilon}\right)\left|\|\phi\|_{L^{\infty}\left(\Omega_{\varepsilon}\right)} \leq C \varepsilon^{\alpha}\right| \log \varepsilon \mid\right\} .
$$

We omit the details. 


\section{Variational reduction and expansion of the energy}

In view of Lemma 3.4, given $\xi=\left(\xi_{1}, \ldots, \xi_{m}\right) \in \mathcal{M}$, we define $\phi\left(\xi^{\prime}\right)$ and $c_{i j}\left(\xi^{\prime}\right)$ to be the unique solution to (63) satisfying (64), where $\xi^{\prime}=\xi / \varepsilon$. We recall the ansatz $V\left(\xi^{\prime}\right)=\sum_{j=1}^{m} b_{j}\left[u_{j}(\varepsilon y)+H_{j}^{\varepsilon}(\varepsilon y)\right]$ defined in (22). Set

$$
\mathcal{F}_{\varepsilon}(\xi)=J_{\varepsilon}\left(V\left(\xi^{\prime}\right)+\phi\left(\xi^{\prime}\right)\right)
$$

where $J_{\varepsilon}$ is the functional defined by

$$
J_{\varepsilon}(v)=\frac{1}{2} \int_{\Omega_{\varepsilon}} a(x)|\nabla v|^{2}-2 \varepsilon^{4} \int_{\Omega_{\varepsilon}} a(x) \cosh v .
$$

Then we have the following results.

Lemma 4.1. If $\xi=\left(\xi_{1}, \ldots, \xi_{m}\right) \in \mathcal{M}$ is a critical point of $\mathcal{F}_{\varepsilon}$ then $v=V\left(\xi^{\prime}\right)+$ $\phi\left(\xi^{\prime}\right)$ is a critical point of $J_{\varepsilon}$, that is, $u=U(\xi)+\tilde{\phi}(\xi)$ is a solution to (1), where $\tilde{\phi}(\xi)(x)=\phi(x / \varepsilon, \xi / \varepsilon)$.

Proof. A direct computation gives

$$
\frac{\partial \mathcal{F}_{\varepsilon}}{\partial \xi_{l}}=\frac{1}{\varepsilon} \frac{\partial J_{\varepsilon}\left(V\left(\xi^{\prime}\right)+\phi\left(\xi^{\prime}\right)\right)}{\partial \xi_{l}^{\prime}}=\frac{1}{\varepsilon} \sum_{i=1}^{2} \sum_{j=1}^{m} c_{i j} \int_{\Omega_{\varepsilon}} \chi_{j} Z_{i j}\left(\frac{\partial V\left(\xi^{\prime}\right)}{\partial \xi_{l}^{\prime}}+\frac{\partial \phi\left(\xi^{\prime}\right)}{\partial \xi_{l}^{\prime}}\right) .
$$

From the assumption $D \mathcal{F}_{\varepsilon}(\xi)=0$ we obtain

$$
\sum_{i=1}^{2} \sum_{j=1}^{m} c_{i j} \int_{\Omega_{\varepsilon}} \chi_{j} Z_{i j}\left(\frac{\partial V\left(\xi^{\prime}\right)}{\partial \xi_{l}^{\prime}}+\frac{\partial \phi\left(\xi^{\prime}\right)}{\partial \xi_{l}^{\prime}}\right)=0, \quad \forall l=1, \ldots, m .
$$

Since $\left\|\partial_{\xi_{l}^{\prime}} \phi\left(\xi^{\prime}\right)\right\|_{L^{\infty}\left(\Omega_{\varepsilon}\right)} \leq C \varepsilon^{\alpha}|\log \varepsilon|^{2 M+3}$ and $\partial_{\xi_{l}^{\prime}} V\left(\xi^{\prime}\right)=(-1)^{l} Z_{i l}+o(1)(i=$ $1,2)$, where $o(1)$ is in the $L^{\infty}$-norm as $\varepsilon \rightarrow 0$, it follows that

$$
\sum_{i=1}^{2} \sum_{j=1}^{m} c_{i j} \int_{\Omega_{\varepsilon}} \chi_{j} Z_{i j}\left((-1)^{l} Z_{i l}+o(1)\right)=0, \quad \forall l=1, \ldots, m,
$$

which is a strictly diagonal dominant system. This implies that $c_{i j}=0, \quad \forall i=$ 1,$2 ; j=1, \ldots, m$.

In order to look for critical points of the function $\mathcal{F}_{\varepsilon}$, a key step is its expected closeness to the function $J_{\varepsilon}(V)$, which we will analyze soon.

Lemma 4.2. The following expansion holds

$$
\mathcal{F}_{\varepsilon}(\xi)=J_{\varepsilon}\left(V\left(\xi^{\prime}\right)\right)+\mathcal{E}_{\varepsilon}\left(\xi^{\prime}\right),
$$

where $\left|\mathcal{E}_{\varepsilon}\right|+\varepsilon^{-1}\left\|\nabla_{\xi^{\prime}} \mathcal{E}_{\varepsilon}\right\| \rightarrow 0$ uniformly on $\mathcal{M}$.

Proof. Let $\mathcal{E}_{\varepsilon}\left(\xi^{\prime}\right)=J_{\varepsilon}(V+\phi)-J_{\varepsilon}(V)$ where $J_{\varepsilon}$ was defined by (67). In order to get the proof of this lemma, we need to show that

$$
\left|\mathcal{E}_{\varepsilon}\right|+\varepsilon^{-1}\left\|\nabla_{\xi^{\prime}} \mathcal{E}_{\varepsilon}\right\|=o(1) .
$$


Using $D J_{\varepsilon}(V+\phi) \cdot \phi=0$, a Taylor expansion and an integration by parts give

$$
\begin{aligned}
& J_{\varepsilon}(V+\phi)-J_{\varepsilon}(V)=-\int_{0}^{1} t D^{2} J_{\varepsilon}(V+t \phi)(\phi, \phi) d t \\
& =-\int_{0}^{1}\left(\int_{\Omega_{\varepsilon}} a(\varepsilon y)[N(\phi)+R] \phi+2 \int_{\Omega_{\varepsilon}} a(\varepsilon y) \varepsilon^{4}[\cosh V-\cosh (V+t \phi)] \phi^{2}\right) t d t,
\end{aligned}
$$

so taking into account that $\|\phi\|_{L^{\infty}\left(\Omega_{\varepsilon}\right)} \leq C \varepsilon^{\alpha}|\log \varepsilon|$, we get

$$
\mathcal{E}_{\varepsilon}=J_{\varepsilon}(V+\phi)-J_{\varepsilon}(V)=O\left(\varepsilon^{2 \alpha}|\log \varepsilon|\right) .
$$

Let us differentiate with respect to $\xi^{\prime}$,

$$
\begin{aligned}
\partial_{\xi^{\prime}}\left[J_{\varepsilon}(V+\phi)-J_{\varepsilon}(V)\right]= & -\int_{0}^{1}\left(\int_{\Omega_{\varepsilon}} \partial_{\xi^{\prime}}[a(\varepsilon y)(N(\phi)+R) \phi]\right. \\
& \left.+2 \int_{\Omega_{\varepsilon}} \partial_{\xi^{\prime}}\left[\varepsilon^{4} a(\varepsilon y)[\cosh V-\cosh (V+t \phi)] \phi^{2}\right]\right) t d t
\end{aligned}
$$

Using the fact that $\left\|\nabla_{\xi^{\prime}} \phi\right\|_{\infty} \leq C \varepsilon^{\alpha}|\log \varepsilon|^{2 M+3}$ and the estimates of the previous sections we get

$$
\partial_{\xi^{\prime}}\left[J_{\varepsilon}(V+\phi)-J_{\varepsilon}(V)\right]=\nabla_{\xi^{\prime}} \mathcal{E}_{\varepsilon}=O\left(\varepsilon^{2 \alpha}|\log \varepsilon|^{2 M+4}\right) .
$$

The continuity in $\xi$ of all these expressions is inherited from that of $\phi$ and its derivatives in $\xi$ in the $L^{\infty}$ norm. The proof is complete.

Now we show the asymptotic expansion of $J_{\varepsilon}(V)$, where $V$ is the approximate solution defined in $(22)$ and $J_{\varepsilon}$ is given by (67).

Lemma 4.3. Let $V$ be given by (22). Then

$$
\begin{aligned}
J_{\varepsilon}(V)= & -16 \pi \sum_{j=1}^{m} a\left(\xi_{j}\right) \log \varepsilon+8 \pi(\log 8-2) \sum_{j=1}^{m} a\left(\xi_{j}\right) \\
& -4 \pi\left[\sum_{j=1}^{m} a\left(\xi_{j}\right) H\left(\xi_{j}, \xi_{j}\right)+\sum_{i \neq j} b_{i} b_{j} a\left(\xi_{j}\right) G\left(\xi_{i}, \xi_{j}\right)\right]+O\left(\varepsilon^{\alpha}\right)
\end{aligned}
$$

uniformly for $\xi=\left(\xi_{1}, \ldots \xi_{m}\right) \in \mathcal{M}$.

Proof. Recall the definition of $V(y)=\sum_{j=1}^{m} b_{j}\left[u_{j}(\varepsilon y)+H_{j}^{\varepsilon}(\varepsilon y)\right]$, we find that it satisfies

$$
-\Delta_{a} V=\varepsilon^{4} \sum_{j=1}^{m} b_{j} e^{u_{j}(\varepsilon y)} \quad \text { in } \Omega_{\varepsilon}, \quad V=0 \quad \text { on } \partial \Omega_{\varepsilon} .
$$

Then we have

$$
\begin{aligned}
\int_{\Omega_{\varepsilon}} a(\varepsilon y)|\nabla V|^{2} & =\varepsilon^{4} \int_{\Omega_{\varepsilon}} a(\varepsilon y)\left(\sum_{i=1}^{m} b_{i} e^{u_{i}(\varepsilon y)}\right) \times\left(\sum_{j=1}^{m} b_{j}\left[u_{j}(\varepsilon y)+H_{j}^{\varepsilon}(\varepsilon y)\right]\right) \\
& =\varepsilon^{4} \sum_{i, j=1}^{m} b_{i} b_{j} \int_{\Omega_{\varepsilon}} a(\varepsilon y) e^{u_{i}(\varepsilon y)}\left[u_{j}(\varepsilon y)+H_{j}^{\varepsilon}(\varepsilon y)\right] .
\end{aligned}
$$


From the Appendix B, it follows that

$$
\begin{aligned}
\int_{\Omega_{\varepsilon}} a(\varepsilon y)|\nabla V|^{2}= & -32 \pi \sum_{j=1}^{m} a\left(\xi_{j}\right) \log \varepsilon+16 \pi(\log 8-1) \sum_{j=1}^{2} a\left(\xi_{j}\right) \\
& -8 \pi\left(\sum_{j=1}^{m} a\left(\xi_{j}\right) H\left(\xi_{j}, \xi_{j}\right)+\sum_{i \neq j} b_{i} b_{j} a\left(\xi_{j}\right) G\left(\xi_{i}, \xi_{j}\right)\right)+O\left(\varepsilon^{\alpha}\right),
\end{aligned}
$$

and

$$
2 \varepsilon^{4} \int_{\Omega_{\varepsilon}} a(\varepsilon y) \cosh V=8 \pi \sum_{j=1}^{m} a\left(\xi_{j}\right)+O\left(\varepsilon^{\alpha}\right) .
$$

This yields our desired result.

\section{Proof of the main results}

In this section we carry out the proofs of our main results.

Proof of Theorem 1.3. Let $\left(\xi_{1}^{*}, \ldots, \xi_{m}^{*}\right)$ be a $C^{0}$-stable critical point for $\mathcal{F}$, by Lemma 4.3 and the definition 1.2 , for any $\varepsilon>0$ small enough, there exists a neighborhood $D_{\varepsilon}^{\prime}$ of $\left(\xi_{1}^{*}, \ldots, \xi_{m}^{*}\right)$, such that the reduced function $\mathcal{F}_{\varepsilon}$ admits at least one critical point in $D_{\varepsilon}^{\prime}$. It follows from Lemma 4.1 that there exist at least one solution for (1). Then the conclusion follows by the ansatz (18).

To prove Theorem 1.1, we will consider a minimization problem. Let us assume that $\bar{x} \in \Omega$ is a strict local minimum point of $a(x)$, i.e., there exists an open subset $B_{\delta}(\bar{x}) \subset \Omega$ such that $\forall y \in B_{\delta}(\bar{x}) \backslash\{\bar{x}\}, a(y)>a(\bar{x})$. In this case, $\mathcal{F}_{\varepsilon}$ reduces to

$$
\begin{aligned}
\mathcal{F}_{\varepsilon}(V)= & -16 \pi\left[a\left(\xi_{1}\right)+a\left(\xi_{2}\right)\right] \log \varepsilon-4 \pi\left[a\left(\xi_{1}\right) H\left(\xi_{1}, \xi_{1}\right)+a\left(\xi_{2}\right) H\left(\xi_{2}, \xi_{2}\right)\right] \\
& +4 \pi\left[a\left(\xi_{1}\right) G\left(\xi_{2}, \xi_{1}\right)+a\left(\xi_{2}\right) G\left(\xi_{1}, \xi_{2}\right)\right]+O(1) .
\end{aligned}
$$

Then we have

Lemma 5.1. For $\varepsilon>0$ small enough, the following minimization problem

$$
\min _{\left(\xi_{1}, \xi_{2}\right) \in \overline{\mathcal{M}}} \mathcal{F}_{\varepsilon}\left(\xi_{1}, \xi_{2}\right)
$$

has a solution in the interior of $\mathcal{M}$.

Proof. Let $\left(\xi_{1}^{\varepsilon}, \xi_{2}^{\varepsilon}\right) \in \overline{\mathcal{M}}$ be the minimizer of $\mathcal{F}_{\varepsilon}$. We need to prove that $\left(\xi_{1}^{\varepsilon}, \xi_{2}^{\varepsilon}\right)$ belongs to the interior of $\mathcal{M}$. First, we will obtain an upper bound. Let

$$
\xi_{j}^{0}=\bar{x}+\frac{1}{\sqrt{|\log \varepsilon|}} \widehat{\xi}_{j}^{0}
$$

where $\widehat{\xi}_{j}^{0} \in \mathbb{R}^{2},\left|\widehat{\xi}_{j}^{0}\right|=\frac{1}{2}, j=1,2$ and $\left|\widehat{\xi}_{1}^{0}-\widehat{\xi}_{2}^{0}\right|=1$. Then it is easy to see $\left(\xi_{1}^{0}, \xi_{2}^{0}\right) \in \mathcal{M}$ since $M=5 / 2 \geq 1$ in this case. From (68) and Lemma 4.2, using 
that $\bar{x}$ is a strict local minimum point of $a$, we obtain

$$
\begin{aligned}
\min \mathcal{F}_{\varepsilon}\left(\xi_{1}, \xi_{2}\right) & \leq-32 \pi a(\bar{x}) \log \varepsilon+16 \pi \sum_{i \neq j} a\left(\xi_{j}^{0}\right) \log \frac{1}{\left|\xi_{j}^{0}-\xi_{i}^{0}\right|}+O(1) \\
& \leq-32 \pi a(\bar{x}) \log \varepsilon+16 \pi a(\bar{x}) \log |\log \varepsilon|+O(1) .
\end{aligned}
$$

Now suppose $\left(\xi_{1}^{\varepsilon}, \xi_{2}^{\varepsilon}\right) \in \partial \mathcal{M}$. There are two possibilities: either there exists a $j_{0}$ such that $\xi_{j_{0}}^{\varepsilon} \in \partial B_{\delta}(\bar{x})$, in which case, $a\left(\xi_{j_{0}}^{\varepsilon}\right) \geq a(\bar{x})+\delta_{0}$ for some $\delta_{0}>0$; or $\left|\xi_{1}^{\varepsilon}-\xi_{2}^{\varepsilon}\right|=|\log \varepsilon|^{-\frac{5}{2}}$.

In the first case, we have

$$
\min \mathcal{F}_{\varepsilon}\left(\xi_{1}, \xi_{2}\right) \geq-16 \pi\left[2 a(\bar{x})+\delta_{0}\right] \log \varepsilon+O(\log |\log \varepsilon|)
$$

which contradicts to $(71)$. This also shows that $a\left(\xi_{j}^{\varepsilon}\right) \rightarrow a(\bar{x})$. By the condition over $a$, we get $\xi_{j}^{\varepsilon} \rightarrow \bar{x}$.

In the second case, we have

$$
\begin{aligned}
\min \mathcal{F}_{\varepsilon}\left(\xi_{1}, \xi_{2}\right) & \geq-32 \pi a(\bar{x}) \log \varepsilon+16 \pi a\left(\xi_{2}^{\varepsilon}\right) \log \frac{1}{\left|\xi_{1}^{\varepsilon}-\xi_{2}^{\varepsilon}\right|}+O(1) \\
& \geq-32 \pi a(\bar{x}) \log \varepsilon+40 \pi a\left(\xi_{2}^{\varepsilon}\right) \log |\log \varepsilon|+O(1)
\end{aligned}
$$

Comparing with (71), we have

$$
40 \pi a\left(\xi_{2}^{\varepsilon}\right) \log |\log \varepsilon|+O(1) \leq 16 \pi a(\bar{x}) \log |\log \varepsilon|+O(1)
$$

which is impossible.

Proof of Theorem 1.1 (completed). According to Lemma 4.1, the function $U(\xi)+\tilde{\phi}(\xi)$, where $U$ is defined by (18) and $\tilde{\phi}$ is defined in Lemma 4.1 , is a solution of problem (1), if we adjust $\xi$ so that it is a critical point of $\mathcal{F}_{\varepsilon}(\xi)$ defined by (66). The Lemma 5.1 then guarantees the existence of a such critical point and thus a solution $u_{\varepsilon}$ for (1). Furthermore, from the ansatz (18), we get that, as $\varepsilon \rightarrow 0, u_{\varepsilon}$ remains uniformly bounded on $\Omega \backslash \cup_{j=1}^{2} B_{\varepsilon^{\alpha}}\left(\xi_{j}^{\varepsilon}\right)$ and

$$
\sup _{B_{\varepsilon^{\alpha}}\left(\xi_{j}^{\varepsilon}\right)}\left|u_{\varepsilon}\right| \rightarrow+\infty, \quad j=1,2 .
$$

The rest of the properties of $u_{\varepsilon}$ can be easily seen from the decomposition of $u_{\varepsilon}$.

\section{Acknowledgments}

The research of the author is partly supported by National Natural Science Foundation of China 10926057, Foundation of Zhejiang Educational Committee under Grant No. Y200908784 and subjects research and development foundation of Hangzhou Dianzi University under Grant No. ZX100204004. 


\section{Appendix A: Proofs of (28) and (29)}

We write $W$ as

$$
\begin{aligned}
W= & \varepsilon^{4} \exp \left(\sum_{j=1}^{m} b_{j}\left(u_{j}+H_{j}^{\varepsilon}\right)\right)+\varepsilon^{4} \exp \left(\sum_{j=1}^{m}\left(-b_{j}\right)\left(u_{j}+H_{j}^{\varepsilon}\right)\right) \\
= & \varepsilon^{4}\left(\frac{8 \mu_{j}^{2}}{\varepsilon^{4}\left(\mu_{j}^{2}+\left|y-\xi_{j}^{\prime}\right|^{2}\right)^{2}}\right)^{b_{j}} \\
& \times \exp \left\{b_{j} H_{j}^{\varepsilon}(\varepsilon y)+\sum_{i \neq j} b_{i}\left(\log \frac{8 \mu_{i}^{2}}{\left(\varepsilon^{2} \mu_{i}^{2}+\varepsilon^{2}\left|y-\xi_{i}^{\prime}\right|^{2}\right)^{2}}+H_{i}^{\varepsilon}(\varepsilon y)\right)\right\} \\
& +\varepsilon^{4}\left(\frac{8 \mu_{j}^{2}}{\varepsilon^{4}\left(\mu_{j}^{2}+\left|y-\xi_{j}^{\prime}\right|^{2}\right)^{2}}\right)^{-b_{j}} \\
& \times \exp \left\{-b_{j} H_{j}^{\varepsilon}(\varepsilon y)+\sum_{i \neq j}\left(-b_{i}\right)\left(\log \frac{8 \mu_{i}^{2}}{\left(\varepsilon^{2} \mu_{i}^{2}+\varepsilon^{2}\left|y-\xi_{i}^{\prime}\right|^{2}\right)^{2}}+H_{i}^{\varepsilon}(\varepsilon y)\right)\right\} .
\end{aligned}
$$

By (20) and Lemma 2.1, we have

$$
\begin{aligned}
H_{i}^{\varepsilon}(\varepsilon y) & =H\left(\varepsilon y, \xi_{i}\right)-\log \left(8 \mu_{i}^{2}\right)+O\left(\varepsilon^{\alpha}\right) \\
& =H\left(\xi_{j}, \xi_{i}\right)-\log \left(8 \mu_{i}^{2}\right)+O\left(\varepsilon^{\alpha}\right)+O\left(\varepsilon^{\alpha}\left|y-\xi_{j}^{\prime}\right|^{\alpha}\right), \quad \forall y \in \Omega_{\varepsilon} .
\end{aligned}
$$

Hence for $\left|y-\xi_{j}^{\prime}\right| \leq \frac{1}{\varepsilon|\log \varepsilon|^{2 M}}$,

$$
\begin{aligned}
b_{j} H_{j}^{\varepsilon} & (\varepsilon y)+\sum_{i \neq j} b_{i}\left[\log \frac{8 \mu_{i}^{2}}{\left(\varepsilon^{2} \mu_{j}^{2}+\left|\varepsilon y-\varepsilon \xi_{i}^{\prime}\right|^{2}\right)^{2}}+H_{i}^{\varepsilon}(\varepsilon y)\right] \\
= & b_{j}\left(H\left(\xi_{j}, \xi_{j}\right)-\log \left(8 \mu_{j}^{2}\right)\right)+\sum_{i \neq j} b_{i}\left[\log \frac{8 \mu_{i}^{2}}{\left|\xi_{j}-\xi_{i}\right|^{4}}+H\left(\xi_{j}, \xi_{i}\right)-\log \left(8 \mu_{i}^{2}\right)\right] \\
& +O\left(\varepsilon^{\alpha}\right)+O\left(\varepsilon^{\alpha}\left|y-\xi_{j}^{\prime}\right|^{\alpha}\right) \\
= & b_{j}\left[H\left(\xi_{j}, \xi_{j}\right)-\log \left(8 \mu_{j}^{2}\right)\right]+\sum_{i \neq j} b_{i} G\left(\xi_{j}, \xi_{i}\right)+O\left(\varepsilon^{\alpha}\right)+O\left(\varepsilon^{\alpha}\left|y-\xi_{j}^{\prime}\right|^{\alpha}\right) \\
= & O\left(\varepsilon^{\alpha}\right)+O\left(\varepsilon^{\alpha}\left|y-\xi_{j}^{\prime}\right|^{\alpha}\right) .
\end{aligned}
$$

The last equality is due to the choice of $\mu_{j}$ in (26). The first equality comes from the expansion of $H_{i}^{\varepsilon}(\varepsilon y)$, the choice of $\xi_{j},(27)$ and

$$
\begin{aligned}
\varepsilon^{2} \mu_{i}^{2}+\varepsilon^{2}\left|y-\xi_{i}^{\prime}\right|^{2} & =\left[\left|\xi_{i}-\xi_{j}\right|+O\left(\left|\varepsilon y-\xi_{j}\right|\right)\right]^{2}+\varepsilon^{2} \mu_{i}^{2} \\
& =\left|\xi_{i}-\xi_{j}\right|^{2} \times\left[1+O\left(\frac{\left|\varepsilon y-\xi_{j}\right|^{2}}{\left|\xi_{i}-\xi_{j}\right|^{2}}\right)+\frac{\varepsilon^{2} \mu_{i}^{2}}{\left|\xi_{i}-\xi_{j}\right|^{2}}\right] \\
& =\left|\xi_{i}-\xi_{j}\right|^{2} \times\left[1+O\left(\varepsilon^{\alpha}\right)+O\left(\varepsilon^{\alpha}\left|y-\xi_{j}^{\prime}\right|^{\alpha}\right)\right] .
\end{aligned}
$$

Therefore, when $\left|y-\xi_{j}^{\prime}\right| \leq \frac{1}{\varepsilon|\log \varepsilon|^{2 M}}$ we obtain

$$
W(y)=\frac{8 \mu_{j}^{2}}{\left(\mu_{j}^{2}+\left|y-\xi_{j}^{\prime}\right|^{2}\right)^{2}}\left[1+O\left(\varepsilon^{\alpha}\right)+O\left(\varepsilon^{\alpha}\left|y-\xi_{j}^{\prime}\right|^{\alpha}\right)\right]+O\left(\varepsilon^{4-\alpha}\right) .
$$


Similarly, for $\left|y-\xi_{j}^{\prime}\right| \leq \frac{1}{\varepsilon|\log \varepsilon|^{2 M}}$ we have

$$
\begin{aligned}
2 \varepsilon^{4} \sinh V= & \varepsilon^{4}\left[\left(\frac{8 \mu_{j}^{2}}{\varepsilon^{4}\left(\mu_{j}^{2}+\left|y-\xi_{j}^{\prime}\right|^{2}\right)^{2}}\right)^{b_{j}}-\left(\frac{8 \mu_{j}^{2}}{\varepsilon^{4}\left(\mu_{j}^{2}+\left|y-\xi_{j}^{\prime}\right|^{2}\right)^{2}}\right)^{-b_{j}}\right] \\
& \times e^{O\left(\varepsilon^{\alpha}\right)+O\left(\varepsilon^{\alpha}\left|y-\xi_{j}^{\prime}\right|^{\alpha}\right)} \\
= & b_{j} \frac{8 \mu_{j}^{2}}{\left(\mu_{j}^{2}+\left|y-\xi_{j}^{\prime}\right|^{2}\right)^{2}}\left[1+O\left(\varepsilon^{\alpha}\right)+O\left(\varepsilon^{\alpha}\left|y-\xi_{j}^{\prime}\right|^{\alpha}\right)\right]+O\left(\varepsilon^{4-\alpha}\right) .
\end{aligned}
$$

On the other hand, for $\left|y-\xi_{j}^{\prime}\right| \geq \frac{1}{\varepsilon|\log \varepsilon|^{2 M}}$ for all $j=1, \ldots, m$, we get easily that

$$
W=O\left(\varepsilon^{4-\alpha}\right), \quad 2 \varepsilon^{4} \sinh V=O\left(\varepsilon^{4-\alpha}\right) .
$$

This, together with (75), implies (29).

Next, by our definitions we get

$$
\begin{aligned}
\Delta_{a(\varepsilon y)} V & =\sum_{j=1}^{m} b_{j}\left[\varepsilon^{2} \Delta_{a(\varepsilon y)} u_{j}(\varepsilon y)+\varepsilon^{2} \Delta_{a(\varepsilon y)} H_{j}^{\varepsilon}(\varepsilon y)\right] \\
& =\sum_{j=1}^{m}\left(-b_{j}\right) \varepsilon^{4} e^{u_{j}(\varepsilon y)}=\sum_{j=1}^{m}\left(-b_{j}\right) \frac{8 \mu_{j}^{2}}{\left(\mu_{j}^{2}+\left|y-\xi_{j}^{\prime}\right|^{2}\right)^{2}} .
\end{aligned}
$$

Therefore, (28) follows from (76) and (77).

\section{Appendix B}

Lemma. For $u_{j}$ and $H_{j}^{\varepsilon}$ are defined respectively by (14) and (19), we have

$$
\begin{aligned}
& \int_{\Omega_{\varepsilon}} a(\varepsilon y) e^{u_{i}(\varepsilon y)}\left[u_{j}(\varepsilon y)+H_{j}^{\varepsilon}(\varepsilon y)\right] \\
& \quad= \begin{cases}-32 \pi a\left(\xi_{j}\right) \log \left(\varepsilon \mu_{j}\right)+8 \pi a\left(\xi_{j}\right) H\left(\xi_{j}, \xi_{j}\right)-16 \pi a\left(\xi_{j}\right)+O\left(\varepsilon^{\alpha}\right) & \text { for } i=j \\
8 \pi a\left(\xi_{i}\right) G\left(\xi_{j}, \xi_{i}\right)+O\left(\varepsilon^{\alpha}\right) & \text { for } i \neq j\end{cases}
\end{aligned}
$$

and

$$
2 \varepsilon^{4} \int_{\Omega_{\varepsilon}} a(\varepsilon y) \cosh V=8 \pi \sum_{j=1}^{m} a\left(\xi_{j}\right)+O\left(\varepsilon^{\alpha}\right) .
$$

Proof. For $i=j$, by the definitions of $u_{j}$ and Lemma (2.2) we obtain

$$
\begin{aligned}
I_{i=j}:= & \varepsilon^{4} \int_{\Omega_{\varepsilon}} a(\varepsilon y) e^{u_{j}(\varepsilon y)}\left[u_{j}(\varepsilon y)+H_{j}^{\varepsilon}(\varepsilon y)\right] \\
= & \int_{\Omega_{\varepsilon}} a(\varepsilon y) \frac{8 \mu_{j}^{2}}{\left(\mu_{j}^{2}+\left|y-\xi_{j}^{\prime}\right|^{2}\right)^{2}}\left(\log \frac{8 \mu_{j}^{2}}{\left(\varepsilon^{2} \mu_{j}^{2}+\left|\varepsilon y-\xi_{j}\right|^{2}\right)^{2}}+H\left(\varepsilon y, \xi_{j}\right)\right. \\
& \left.-\log \left(8 \mu_{j}^{2}\right)+O\left(\varepsilon^{\alpha}\right)+O\left(\varepsilon^{\alpha}\left|y-\xi_{j}^{\prime}\right|^{\alpha}\right)\right) .
\end{aligned}
$$


Changing variables $\mu_{j} z=y-\xi_{j}^{\prime}$ and denoting by $\omega_{j}=\left\{z \in \mathbb{R}^{2} ; \xi_{j}+\varepsilon \mu_{j} z \in \Omega\right\}$, we have

$$
I_{i=j}=\int_{\omega_{j}} \frac{8 a\left(\xi_{j}+\varepsilon \mu z\right)}{\left(1+|z|^{2}\right)^{2}}\left[\log \frac{1}{\left(1+|z|^{2}\right)^{2}}+H\left(\xi_{j}+\varepsilon \mu z, \xi_{j}\right)-4 \log \left(\varepsilon \mu_{j}\right)\right]+O\left(\varepsilon^{\alpha}\right) .
$$

Note the facts that

$$
\int_{\omega_{j}} a\left(\xi_{j}+\varepsilon \mu_{j} z\right) \frac{8}{\left(1+|z|^{2}\right)^{2}}=8 \pi a\left(\xi_{j}\right)+O\left(\varepsilon \mu_{j}\right)
$$

and

$$
\int_{\omega_{j}} \frac{8}{\left(1+|z|^{2}\right)^{2}}\left|H\left(\xi_{j}+\varepsilon \mu_{j} z, \xi_{j}\right)-H\left(\xi_{j}, \xi_{j}\right)\right|=O\left(\varepsilon^{\alpha}\right),
$$

we can get

$$
I_{i=j}=-32 \pi a\left(\xi_{j}\right) \log \left(\varepsilon \mu_{j}\right)+8 \pi a\left(\xi_{j}\right) H\left(\xi_{j}, \xi_{j}\right)-16 \pi a\left(\xi_{j}\right)+O\left(\varepsilon^{\alpha}\right) .
$$

For $i \neq j$, a calculation similar to (75) shows that

$$
\begin{aligned}
\int_{\Omega_{\varepsilon}} & a(\varepsilon y) e^{u_{i}(\varepsilon y)}\left[u_{j}(\varepsilon y)+H_{j}^{\varepsilon}(\varepsilon y)\right] \\
\quad= & \int_{\omega_{i}} \frac{8 a\left(\xi_{i}+\varepsilon \mu_{i} z\right)}{\left(1+|z|^{2}\right)^{2}}\left[\log \frac{1}{\left|\xi_{i}-\xi_{j}\right|^{4}}+H\left(\xi_{i}, \xi_{j}\right)\right]+O\left(\varepsilon^{\alpha}\right) \\
\quad= & 8 \pi a\left(\xi_{i}\right) G\left(\xi_{j}, \xi_{i}\right)+O\left(\varepsilon^{\alpha}\right) .
\end{aligned}
$$

Therefore, taking account of (26) we derive

$$
\begin{aligned}
\int_{\Omega_{\varepsilon}} a(\varepsilon y)|\nabla V|^{2}= & -32 \pi \sum_{j=1}^{m} a\left(\xi_{j}\right) \log \varepsilon+16 \pi(\log 8-1) \sum_{j=1}^{m} a\left(\xi_{j}\right) \\
& -8 \pi\left[\sum_{j=1}^{m} a\left(\xi_{j}\right) H\left(\xi_{j}, \xi_{j}\right)+\sum_{i \neq j} b_{i} b_{j} a\left(\xi_{j}\right) G\left(\xi_{i}, \xi_{j}\right)\right]+O\left(\varepsilon^{\alpha}\right) .
\end{aligned}
$$

Next, a simple computation yields

$$
2 \varepsilon^{4} \int_{\Omega_{\varepsilon}} a(\varepsilon y) \cosh V=2 \varepsilon^{4} \sum_{j=1}^{m} \int_{B_{\frac{\delta}{\varepsilon}}} a(\varepsilon y) \cosh V+O\left(\varepsilon^{2}\right) .
$$

Suppose $j$ is odd first. Then

$$
\begin{aligned}
& 2 \varepsilon^{4} \int_{B_{\frac{\delta}{\varepsilon}}\left(\xi_{j}^{\prime}\right)} a(\varepsilon y) \cosh V=\varepsilon^{4} \int_{B_{\frac{\delta}{\varepsilon}}\left(\xi_{j}^{\prime}\right)} a(\varepsilon y) e^{V}+O\left(\varepsilon^{\alpha}\right) \\
& =\varepsilon^{4} \int_{B_{\frac{\delta}{\varepsilon}}\left(\xi_{j}^{\prime}\right)} a(\varepsilon y) e^{u_{j}(\varepsilon y)} e^{H_{j}^{\varepsilon}+\sum_{l \neq j} b_{l}\left(u_{l}+H_{l}^{\varepsilon}\right)}+O\left(\varepsilon^{\alpha}\right)=8 \pi a\left(\xi_{j}\right)+O\left(\varepsilon^{\alpha}\right) .
\end{aligned}
$$

Therefore, we have

$$
2 \varepsilon^{4} \int_{B_{\frac{\delta}{\varepsilon}}\left(\xi_{j}^{\prime}\right)} a(\varepsilon y) \cosh V=8 \pi a\left(\xi_{j}\right)+O\left(\varepsilon^{\alpha}\right) .
$$


Similarly for $j$ even, we also have (79). So we get

$$
\varepsilon^{4} \int_{\Omega_{\varepsilon}} a(\varepsilon y)\left(e^{V}+e^{-V}\right)=8 \pi \sum_{j=1}^{m} a\left(\xi_{j}\right)+O\left(\varepsilon^{\alpha}\right) .
$$

The lemma follows.

\section{References}

[1] Bartolucci, D., Pistoia, A.: Existence and qualitative properties of concentrating solutions for the sinh-Poisson equation. IMA J. Appl. Math. 72(6), 706-729 (2007)

[2] Bates, P., Dancer, E.N., Shi, J.: Multi-spike stati onary solutions of the CahnHilliard equation in higher-dimension and instability. Adv. Differ. Equ. 4, 1-69 (1999)

[3] Chen, C.C., Lin, C.S.: Sharp estimates for solutions of multi-bubbles in compact Riemann surfaces. Commun. Pure Appl. Math. 55(6), 728-771 (2002)

[4] Del Pino, M., Felmer, P., Musso, M.: Two-bubble solutions in the super-critical Bahri-Coron's problem. Calc. Var. Partial Differ. Equ. 16, 113-145 (2003)

[5] Del Pino, M., Kowalczyk, M., Musso, M.: Singular limits in Liouville-type equations. Calc. Var. Partial Differ. Equ. 24, 47-81 (2005)

[6] Dancer, E.N., Yan, S.: Multipeak solutions for a singular perturbed Neumann problem. Pac. J. Math. 189, 241-262 (1999)

[7] Esposito, P., Grossi, M., Pistoia, A.: On the existence of blowing-up solutions for a mean field equation. Ann. Inst. H. Poincaré Anal. Nonlinéaire 22, 227$257(2005)$

[8] Esposito, P., Pistoia, A., Wei, J.C.: Concentrating solutions for the Hénon equation in $\mathbb{R}^{2}$. J. Anal. Math. 100, 249-280 (2006)

[9] Esposito, P., Wei, J.C.: Non-simple blow-up solutions for the Neumann twodimensional sinh-Gordon equation. Calc. Var. Partial Differ. Equ. 34, 341$375(2009)$

[10] Flierl, G.R.: Isolated eddy models in geophysics. Annu. Rev. Fluid Mech. 19, 493-530 (1987)

[11] Gui, C., Wei, J.: Multiple interior spike solutions for some singular perturbed Neumann problems. J. Differ. Equ. 158, 1-27 (1999)

[12] Gui, C., Wei, J.: On multiple mixed interior and boundary peak solutions for some singularly perturbed Neumann problems. Can. J. Math. 52, 522-538 (2000)

[13] Gui, C., Wei, J., Winter, M.: Multiple boundary peak solutions for some singularly perturbed Neumann problems. Ann. Inst. H. Poincaré Anal. Nonlinéaire 17, 249-289 (2000) 
[14] Hopfinger, E.J., Van Heijst, G.J.G.: Vortices in rotating fluids. Annu. Rev. Fluid Mech. 25, 241-289 (1993)

[15] Jost, J., Wang, G.F., Ye, D., Zhou, C.Q.: The blow up analysis of solution to the elliptic sinh-Gordon equation. Calc. Var. Partical Differ. Equ. 31, 263-276 (2008)

[16] Marteau, D., Cardoso, O., Tabeling, P.: Equilibrium states of twodimensional turbulence: an experimental study. Phys. Rev. E 51, 5124-5127 (1995)

[17] Ohtsuka, H., Suzuki, T.: Mean field equation for the equilibrium turbulence and a related functional inequlity. Adv. Differ. Equ. 11(3), 281-304 (2006)

[18] Rey, O., Wei, J.: Blow-up solutions for an elliptic Neumann problem with subor-supcritical nonlinearity, I: $N=3$. J. Funct. Anal. 212(2), 472-499 (2004)

[19] Spruck, J.: The elliptic sinh Gordon equation and the construction of toroidal soap bubbles. In: Calculus of Variations and Partial Differential Equation (Trento, 1986). Lecture Notes in Mathematics, vol. 1340, pp. 275-301. Springer, Berlin (1988)

[20] Wei, J., Wei, L., Zhou, F.: Concentrating Solutions for some Neumann Problem with Equilibrium Vortices. Preprent

[21] Wei, J., Ye, D., Zhou, F.: Bubbling solutions for an anisotropic Emden-Fowler equation. Cal. Var. and Partial Differ. Equ. 28, 217-247 (2007)

[22] Wei, L.: On the number of nodal bubbling solutions to a sinh-Poisson equation. Houston J. Math. 35(1), 291-326 (2009)

[23] Wente, H.: Conterexample to a conjecture of H. Hopf. Pac. J. Math. 121, 193243 (1986)

Long Wei

Institute of Applied Mathematics and Engineering Computations,

Hangzhou Dianzi University,

Zhejiang 310018,

China

e-mail: alongwei@gmail.com

Received: 12 September 2010.

Accepted: 10 April 2011. 